\title{
Anodizing under conditions of oxygen activation of the inter-electrode gap
}

\author{
Nikolay Kolenchin ${ }^{1}$ and Denis Denisenko ${ }^{1, *}$ \\ ${ }^{1}$ Tyumen Industrial University, Tyumen, Russian Federation, 625000
}

\begin{abstract}
Structured alumina is currently used in a wide range of applications. Interest in a surface with a wear-resistant coating motivates creation of methods for high-speed oxidation with an increase in the thickness and hardness of the layer, with obligatory observance of environmental parameters and a decrease in the energy intensity of production. Considering the activity of aluminum towards oxygen, a very important aspect is the search for conditions to increase the natural oxide film to the level of functional significance. The generally accepted scheme of classical anodizing represents a closed system of an electrolytic cell, inside which the elements are activated in the interelectrode gap to the state of ionic excitation under the action of an electric field. The efficiency of interaction depends on the medium's nature and variability of the voltampere parameters. This work proposes a different mechanism for intensifying the process. Oxygen is activated outside the electrolytic cell and in the allotropic state, in the form of ozone, is transmitted into the interelectrode gap. The phase composition, structure, thickness, and microhardness are investigated. The aim of the research is to establish the effect of ozone on the oxidation process.
\end{abstract}

\section{Introduction}

The history of growing oxide on an aluminum surface has a century and a half of experience with a huge baggage of various technological solutions that determine functionality of the formed layer. Film oxide can be conditionally divided into two types: with a clearly defined structure of hexagonal honeycombs with a hole in the middle and oxide with an unregulated shape. The structure formation is determined by the applied voltage across the electrodes [1]. The method of structural oxide formation on the surface of aluminum alloys without sparking and micro-breakdowns is commonly called anodizing. The process of structureless molding with formation of sparks and micro-breakdowns on the anode surface is referred to as plasmaelectrolytic or micro-arc oxidation [2].

Interest in structured oxide is due to the ability to control its main parameters geometry, phase state, rate of formation, which ultimately determines the functional set of the formed film [3].

The kinematic scheme of anodizing is considered as two, simultaneously occurring processes - formation and dissolution of oxide $[4,5]$. Depending on the functional purpose

*Corresponding author: ardiasardias@gmail.com 
of the surface, a task is formed to select the factors that ensure a harmonious ratio of growth and dissolution rates. The aggressiveness of the medium is primarily determined by the nature of the electrolyte, and its gradient is a function of temperature [6]. The most widely used as a working medium are aqueous solutions of sulfuric, phosphoric, oxalic acids, on the basis of which a line of liquids with more complex chemical composition is formed.

Variability of the superposition of the electric field is reflected in:

1. Geometrical parameters of the structure - pore and cell size.

2. The phase state of the oxide - amorphous, crystalline, and modification.

Regardless of the number of participants in the electrochemical process and conditions of their interaction, there are several variants of redox reactions involving oxygen-containing anions.

It is generally accepted that the main donors of oxygen are water $\mathrm{H}_{2} \mathrm{O}$ and hydroxide ions $\mathrm{OH}^{-}$. Participation of oxygen of the acid residue is also not excluded, but the main role is assigned to it as a sculptor of individual elements of the oxide structure. Priority in the chemistry of the process has not yet been unambiguously established and is determined by the level of cations and anions activation, their concentration at the metal-oxide interface. This is noted by a number of researchers. When developing a holistic model of stationary growth of anodic aluminum oxide [7], the authors found that the growth rate of the oxide depends on the amount of $\mathrm{O}^{2-}$ near the metal-oxide interface. The developers of the anodizing method explain the increase in the rate of oxide formation by ionization of oxygen donors [8]. By placing the anode at the interface between the liquid and air phases, and the cathode above the electrolyte, when an electric field is applied at the moments of discharge phenomena, a funnel is formed, filled with ionized anions of the electrolyte, water, and oxygen.

Anodizing in less aggressive solutions with various ionic additives [9] enables one to increase the rate of oxide growth.

The self-ordered format of pore formation depends on the rate of water dissociation and transfer of oxygen anions to the interaction zone with aluminum [10]. Investigating the design features of the oxide formed under the conditions of a mild anodizing regime, it was found that only $14.4 \%$ of aluminum participates in the oxidation process. The rest goes into solution.

Thus, the proposed options for increasing the efficiency of aluminum utilization in the pore space are associated with the expansion of ionic composition of the anions at the metaloxide interface or the tightening of the cooling regime. This allows us to assume some selectivity of the course of redox reactions in the active zone, as well as to judge the shift in the balance of the growth rates and dissolution of the oxide in favor of the former.

This work presents the results of studies of the formation of an ordered oxide with the dominance of active oxygen.

\section{Research}

Anodizing was carried out in a galvanostatic mode in a sulfuric acid electrolyte with a sulfuric acid concentration of $10 \mathrm{wt} \%$. The temperature of the solution was $10{ }^{\circ} \mathrm{C}$ and was automatically maintained by a refrigeration unit. The samples in the amount of 5 pieces measuring 20x30x3mm were made of D16 deformed alloy. The electrolyte was bubbled with an ozone-air flow supplied through a porous packing at a rate of $2 \mathrm{l} / \mathrm{min}$. The current density was $5 \mathrm{~A} / \mathrm{dm}^{2}$. The duration of the anodizing process was $30 \mathrm{~min}$. The samples were preliminarily defatted in ethanol heated to $70^{\circ}$, followed by rinsing in water. An ozone-air mixture was passed through the electrolyte for 15 minutes before the voltage was applied. The ozone concentration in the air flow was $5 \mathrm{mg} / \mathrm{l}$. The flow rate was controlled by a rotameter. The concentration of ozone in the air flow was monitored with an ozonometer. 
Transport lines for supplying ozone-air mixture were made of glass and stainless tubes. The air was blown in with a compressor. The cathode was a Kh18N10T stainless steel plate.

The chemical composition of the surface layer at a depth of $10 \mathrm{~nm}$ was determined by the XPS method. The results are shown in Figure 1. Using the Specs Lab database, the concentration of elements in atomic percentages was determined, Table 1.

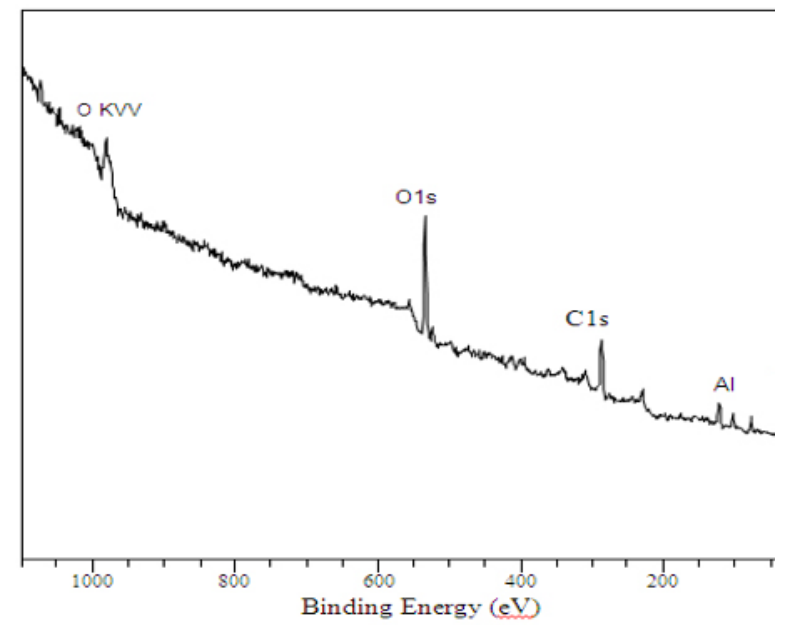

Fig. 1. Spectra of elements at a depth of $5 \mathrm{~nm}$

Table 1. Concentration of elements in at. \%

\begin{tabular}{|c|c|c|c|c|c|}
\hline Sample & Depth, $\mathrm{nm}$ & $\mathrm{O}$ & $\mathrm{Al}$ & $\mathrm{S}$ & $\mathrm{C}(\mathrm{O}) / \mathrm{C}(\mathrm{met})$ \\
\hline \multirow{3}{*}{ D16 } & 0 & 36.5 & 9.6 & $\mathrm{TA}^{*}$ & 3.8 \\
\cline { 2 - 6 } & 0.5 & 32.7 & 16.0 & $\mathrm{TA}^{*}$ & 2.04 \\
\cline { 2 - 6 } & 5 & 33.1 & 21.7 & $\mathrm{TA}^{*}$ & 1.53 \\
\hline \multirow{3}{*}{$\begin{array}{c}\text { Model } \\
\text { Al }\end{array}$} & 0 & 33.1 & 19.9 & 0 & 1.67 \\
\cline { 2 - 6 } & 0.5 & 45.3 & 47.7 & 0 & 0.95 \\
\cline { 2 - 6 } & 6.5 & 7.9 & 87.6 & 0 & 0.09 \\
\hline \multicolumn{7}{|c|}{ *TA - in trace amounts } \\
\hline
\end{tabular}

The values in the right-hand column determine the stoichiometry of the aluminumoxygen compound. In deep layers, it corresponds to aluminum oxide, since their ratio is 1.5.

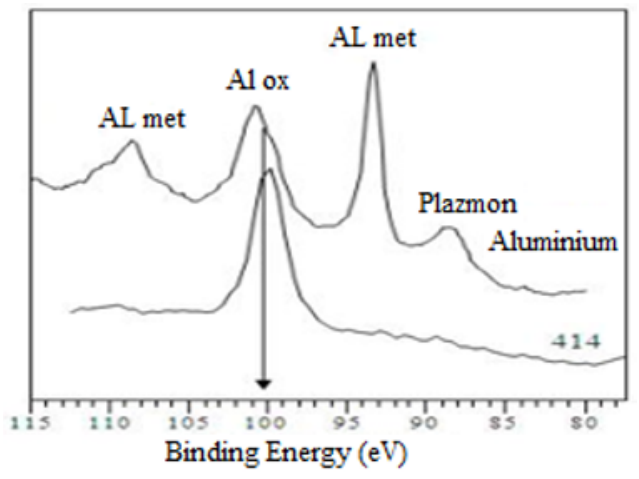

Fig 2. Spectroscopy results at a depth of $5 \mathrm{~nm}$ 
The results of Auger spectroscopy of the surface layer, presented in Figure 2, confirm formation of $\mathrm{Al}_{2} \mathrm{O}_{3}$. An insignificant content of sulfur, manifested in the spectra, is the result of introduction of acid residue anions into the oxide structure.

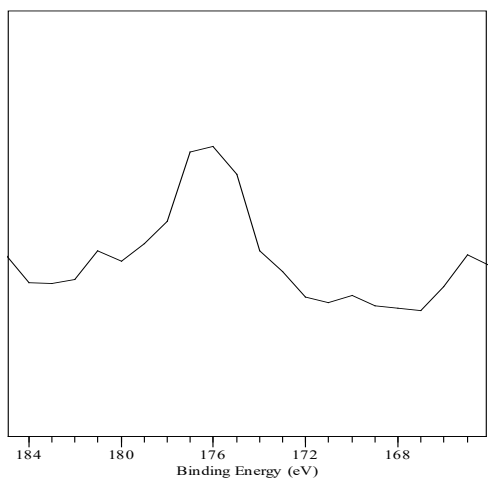

Fig. 3. Binding energy of sulfur at a sample depth of $0.5 \mathrm{~nm}$

An X-ray spectral microanalyzer investigated the composition of elements at a depth of $1 \mu \mathrm{m}$, which confirmed the presence of sulfur. An insignificant spike in carbon concentration should be regarded as a result of sample etching. The rest of the elements are in the substrate and do not participate in oxide formation.

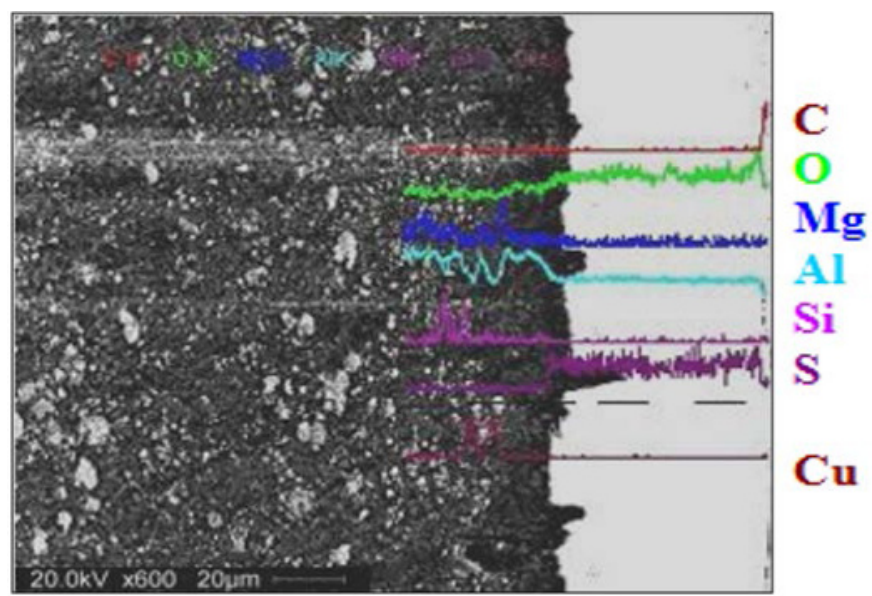

Fig. 4. Surface spectrogram at a depth of $1 \mu \mathrm{m}$.

The phase composition was determined using a D8 Advance diffractometer in $\mathrm{CuK}_{\alpha}$ radiation 


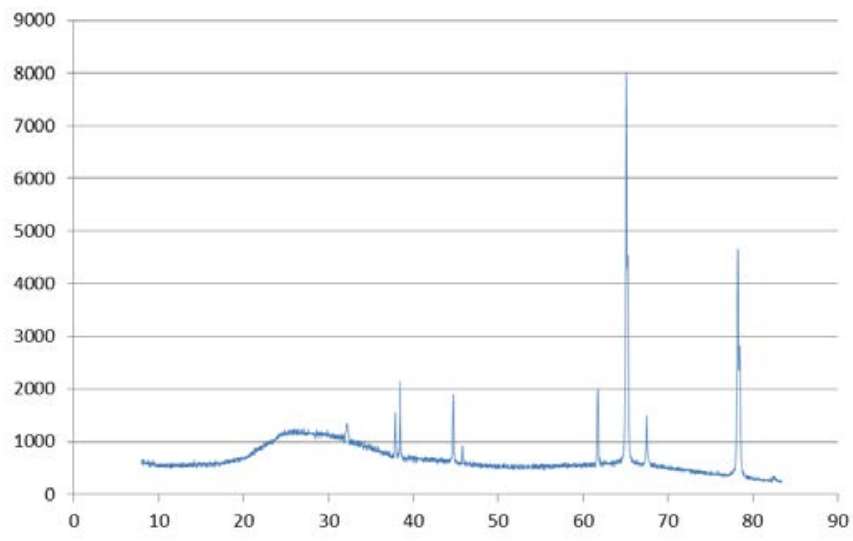

Fig. 5. X-ray diffraction pattern of the formed oxide

Figure 5 shows an X-ray diffraction pattern of the anode layer, in which the amorphous and crystalline components are clearly visible. The amorphous phase is defined in the range of angles $20-40^{\circ}$. Presence of the crystalline phase is confirmed by bursts of intensity at angles: $37.77^{\circ}, 45.95^{\circ}, 60.95^{\circ}$. According to the PDF-2 (ISCD) phase bank, the modification corresponds to $\gamma-\mathrm{Al}_{2} \mathrm{O}_{3}$.

The microstructural surfaces were examined using Integra Aura - an atomic force probe microscope. The results are shown in Figure 6.

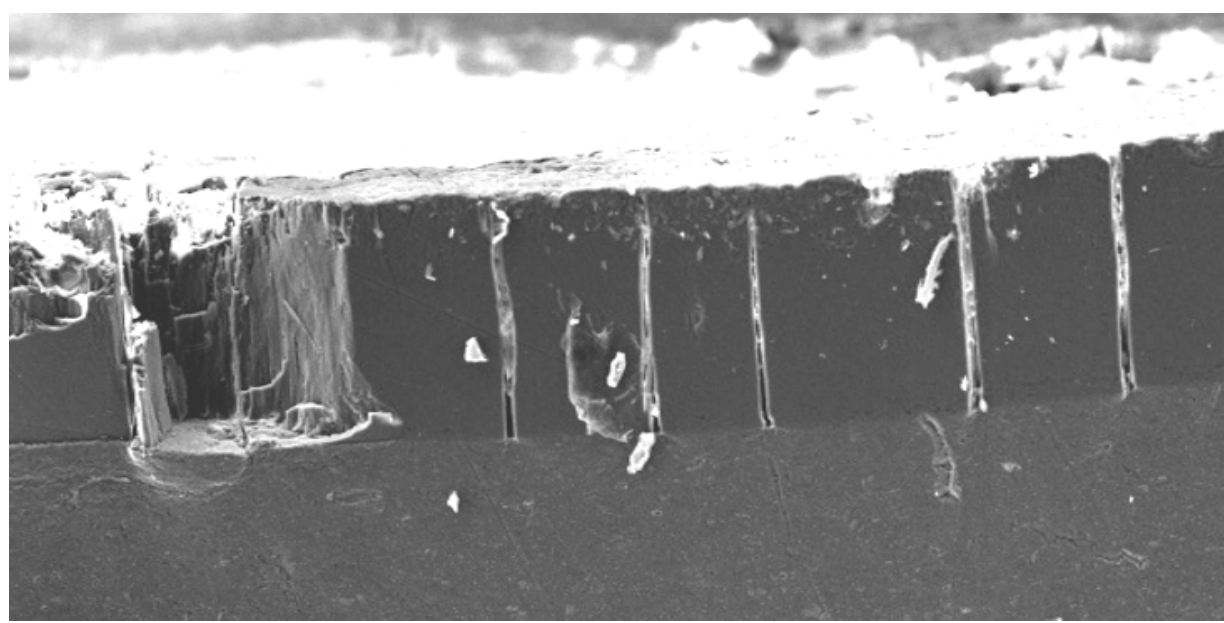

Fig 6. Cross-sectional microstructure at $95 \mathrm{x}$ magnification

The studies of the cross section of the oxide film have shown that, along with the pores with a dimension of (10-150) nm, characteristic of conventional anodizing, there are larger channels, which are tens of times larger in diameter than nanostructured objects.

The thickness of the oxide layer was determined by the express method using ConstantK5 - an ultrasonic contact thickness gauge. In half an hour, a layer $57 \mu \mathrm{m}$ thick was formed. The value is derived from five measurements.

The microhardness was determined with a PMT-3 device by indenting a diamond pyramid with a load of $20 \mathrm{~g}$. From five measurements, the average microhardness value was determined, which corresponded to $6100 \mathrm{MPa}$. 


\section{Discussion of the results}

Considering that ozone is the strongest oxidizing agent and its activity can be directed to all potential participants in the electrochemical process, it was very important to establish formation of aluminum oxide. Auger spectroscopy shows the presence of oxide in the film, and this proves participation of the ozone itself or atomic oxygen, which appears as a result of decomposition of the former.

The presence of sulfur in the entire volume of the coating, detected by Auger spectroscopy, proves incorporation of the anion of the acid residue into the structure of the film and is in good agreement with previous studies [11].

The process of ozone decomposition into molecular and atomic oxygen is inevitable, since the metal-oxide interface is a zone of Joule heat release during discharge phenomena. A rise in temperature by several degrees increases the rate of ozone decay by several orders of magnitude [12]. An increase in the ionic density in the reaction zone can be judged on the rate of oxide formation. In earlier experiments, it was found that anodizing under the same conditions, but without ozone, formed a film with a thickness of $40 \mu \mathrm{m}$ in $30 \mathrm{~min}$, and the microhardness corresponded to a value of $4450 \mathrm{MPa}$. The increase in hardness is most likely due to the appearance of $\gamma-\gamma-\mathrm{Al}_{2} \mathrm{O}_{3}$ crystalline modification. Crystal formation can be explained by an exothermic process in the nanovolume of a pore at the moment of ozone decomposition. It is known that the decomposition of 2 moles of gas is accompanied by the release of $297.4 \mathrm{~kJ}$ of heat. Most likely, this is sufficient for partial crystallization of the oxide, the appearance of which was confirmed by X-ray structural studies.

Of particular interest is the appearance of filamentous megachannels against the background of the nanoscale pore geometry. The appearance of submillimeter-scale pores or bowl-shaped pores during anodizing in sulfuric acid electrolytes was noted by a number of researchers $[13,14]$ and is explained by the appearance of electroconvective vortices of a larger radius at the anode surface with a corresponding temperature rise followed by strong electrochemical etching. Formation of the embryo of the filamentous channel can occur at the moment of ozone decomposition on the anode surface with the release of heat and an increase in the aggressiveness of the acid anions. The absence of a dielectric barrier in a short period of time causes an increase in the ion yield of aluminum in the formed hole and attraction of oxygen-containing anions. The alternating decays of molecular ozone increase the rate of oxide formation and maintain the pore profile throughout the entire anodizing process.

\section{Conclusions}

1. The introduction of ozone into the electrolytic solution can be regarded as a step towards intensification of the oxidation process.

2. The exothermic nature of ozone decomposition at the metal-electrolyte and metal-oxide interface contributes to the crystallization of phase oxides and formation of large transport arteries in the film microstructure.

3. The proposed scheme of activating oxygen-containing anions is an environmentally friendly measure for increasing the efficiency of a protective layer deposition.

\section{References}

1. W. Lee, U. Gösele, K. Nielsch, Nat. Mater., 5, pp. 741-747 (2016).

2. W. Gu, G. Lu, H. Chen. Mater. Sci. and Eng., 44, pp. 158-162 (2017).

3. G. Schmidt. J. Mater. Chem., 5, pp. 1231-1238 (2012) 
4. G. Thompson, G. Wood. Nat., 29, pp. 230-232 (1983).

5. B. Tran. Amer. Ceram. Bull., 6, pp. 563-568 (1977).

6. G. Sulka, K. Parkola, Th. Sol. Film., 51, pp. 338-345 (2006).

7. G. Patermarakis. Electrochim. Acta, 9, pp. 2434-2443 (2009).

8. E. Averyanov, Mosc. Rad., 4 pp. 416-423 (1983)

9. M. Salerno, N. Patra, Mater Lett, 63, pp. 1826 (2009).

10. Z. Su, G. Hähner, J. Mater. Chem., 18, pp. $5787-5795$ (2008).

11. G. Patermarakis, J. Electroan. Chem., 2, pp. 25-41, (2015).

12. V.V. Lunin. Physical chemistry of ozone (Publ. MGU, Moscow, 1998).

13. M. Pashchanka, J Mater. Chem., 2, p.15867-15875 (2002).

14. M. Moutarliera, B. Gigandeta Corr. Sci.6 4, pp. 937-951(2005). 